\title{
Recurrent Pleomorphic Adenoma of the Parotid Gland
}

\author{
Soo Yeon Park, \\ Ki-Taik Han, \\ Min-Cheol Kim, \\ Jin Soo Lim
}

Department of Plastic and Reconstructive Surgery, St. Vincent's Hospital, College of Medicine, The Catholic University of Korea, Suwon, Korea

No potential conflict of interest relevant to this article was reported.
Pleomorphic adenoma is the most common type of salivary gland tumor and the most common tumor of the parotid gland. Because of its propensity for invasion, pleomorphic adenoma of the parotid requires superficial parotidectomy or total parotidectomy to minimize the risk of tumor recurrence. We report a case of pleomorphic adenoma of the parotid gland with repeated recurrences. A 23-year-old male patient presented with a protruding neck mass. Six years prior to this presentation, the patient had undergone superficial parotidectomy for the removal of pleomorphic adenoma of the right parotid gland at our institution. The patient experienced recurrence at 17 months after the initial resection, which required a total parotidectomy with partial resection of the facial nerve. Pathologic examination revealed histologic findings consistent with pleomorphic adenoma across the surgical specimen from all three of the operations. The patient suffered from facial nerve paralysis, with facial expressions partially recovered over a year.

Keywords: Recurrent pleomorphic adenoma / Salivary gland tumor / Superficial parotidectomy / Total parotidectomy

\section{INTRODUCTION}

Pleomorphic adenoma is the most common tumor of the parotid gland. Enucleation alone is associated with high recurrence rates ( $8 \%-45 \%)$, owing to the invasive histopathologic characteristics of the tumor. In contrast, total parotidectomy is associated with a much lower rate of recurrence at $0.4 \%[1,2]$.

The present report describes a case of pleomorphic adenoma that recurred twice.

\section{CASE REPORT}

A 23-year-old male patient presented with a protruding neck mass. Six years prior to this presentation, the patient had undergone superficial parotidectomy for the removal of pleomorphic adenoma of the right parotid gland at our institution (Fig. 1). The patient ex-

\section{Correspondence: Jin Soo Lim}

Department of Plastic and Reconstructive Surgery, St. Vincent's Hospital,

College of Medicine, The Catholic University of Korea, 93 Jungbu-daero, Paldalgu, Suwon 16247, Korea

E-mail: prsdrlim@yahoo.com

Received April 6, 2016 / Revised May 24, 2016 / Accepted May 25, 2016 perienced recurrence at 17 months after the initial resection, which required a total parotidectomy.

At physical examination, the $1.5 \mathrm{~cm}$ mass was located within the surgical scar on the upper portion of the sternocleidomastoid muscle. It was firm, indolent, and nontender. Computed tomography (CT) scan of the neck revealed a 5.1×3.2-cm multinodular mass at the site of initial tumor location, along the $1.5-\mathrm{cm}$ subcutaneous mass in the surgical scar area (Fig. 2).

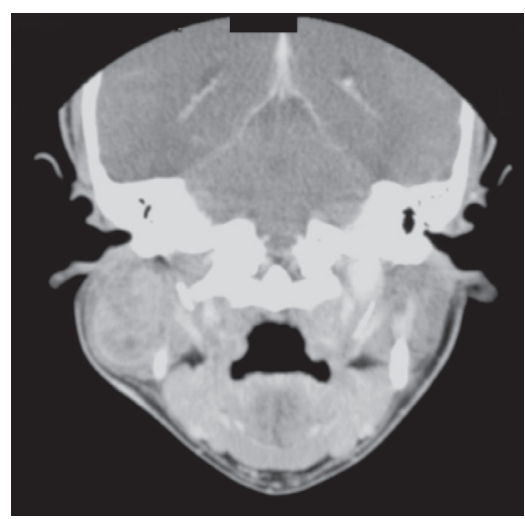

Fig. 1. At the time of first recurrence, computed tomography scan shows a $3.0 \times 3.5-\mathrm{cm}$ heterogeneous mass in the superficial lobe of the right parotid gland. 


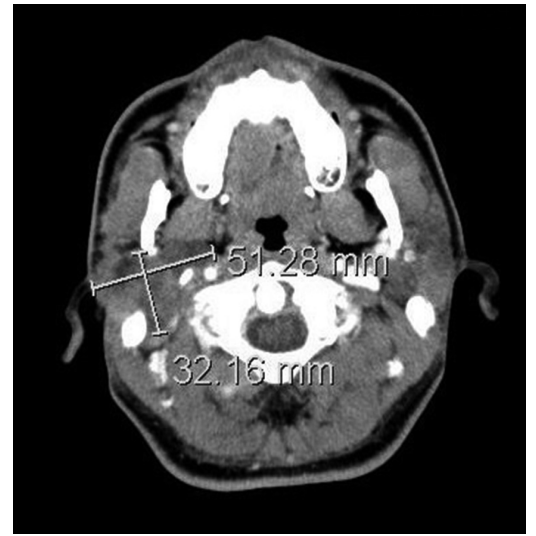

Fig. 2. Computed tomography scan at second recurrence (five years later). The right parotid mass extends into the subcutaneous layer of the right side of the neck and the upper portion of the sternocleidomastoid muscle.

Under general anesthesia, the mass was excised with wide margins. At the site of cervical surgery scar, the mass was located in the subcutaneous layer and surrounded by fibrotic tissue, but no glandular tissue was observed. The multinodular mass deeply rooted in the trunk of facial nerve and expanded beyond the area occupied by the first tumor. Intraoperative frozen section confirmed both masses having benign histology. Therefore, both masses were resected along with all surrounding scar tissues. A portion of the frontal branch of facial nerve were sacrificed for complete resection of the parotid mass.

Histopathologic examination of the initial resection demonstrated solitary nodules containing a mixture of epithelial and mesenchymal structures. It was a typical pleomorphic adenoma with tubular or linear architecture of epithelial cells and hyaline and myxochondroid appearance of stromal components. Tissues obtained after the second surgery also comprised multiple nodules. Histopathologic findings indicated that the recurrent tumor was also a typical pleomorphic adenoma but composed of more than 80 large and small nodules. We observed both the presence of microscopic extensions or pseudopodia-finger-like expansions-protruding beyond pseudocapsule and capsular penetration. Tissues obtained after the third operation, conducted five years following the second, consisted of more than 50 nodules, similar to that in the tissues collected after the second surgery. Histologic findings were identical for surgical specimen from initial, second, and third opera- tion - except that one of the nodules contained mostly epithelial cells with high cellularity and few mitotic figures (1 mitotic figure per 10 high power fields) (Fig. 3). Immediately after the third operation, the patient experienced right-sided facial paralysis, as expected from the manipulation and partial resection of the facial nerve. At 18-month follow-up, we observed an improvement in facial movement compared with the state immediately after the surgery. However, the patient continued to experience difficulty making facial expressions. A follow-up CT study did not show any evidence of recurrence (Fig. 4).

\section{DISCUSSION}

Pleomorphic adenoma is the most common benign tumor of the parotid gland. Most instances of the adenoma occur in individuals between 30 and 60 years of age, with a predilection for the female
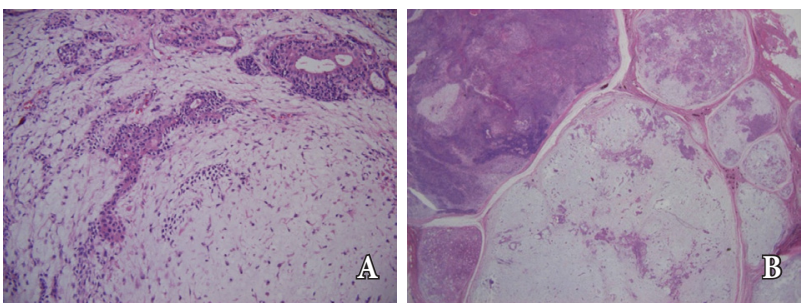

Fig. 3. Histology of the tumor at each recurrence. (A) The specimen from the first recurrence contained tubulo-ductal structures surrounded by myxochondroid matrix typical for pleomorphic adenoma of the parotid gland $(\mathrm{H} \& \mathrm{E}, \times 100)$. (B) The specimen from the second recurrence was histologically similar to the first specimen, with shows multi-nodular pleomorphic adenoma and the nodule with high cellularity and few mitotic figures $(\mathrm{H} \& \mathrm{E}, \times 40)$.

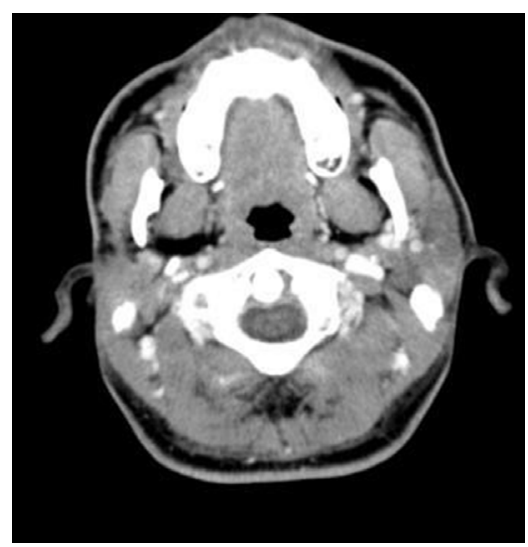

Fig. 4. Computed tomography scan shows no evidence of tumor recurrence in the right parotid region at 18 months after the third operation. 
sex. Histologically, the tumor consists of epithelial and mesenchymal cells, but various patterns may be present in any given tumor [3]. Clinically, pleomorphic adenoma presents as a slow-growing painless tumor, which becomes malignant in $2 \%-24 \%$ of the cases $[3,4]$.

Pleomorphic adenoma is characterized by the presence of microscopic extensions protruding beyond pseudocapsule or by capsular penetration. Simple enucleation of pleomorphic adenoma is associated with high recurrence rates, between $8 \%$ and $45 \%$, which is reduced to less than 5\% with superficial parotidectomy and further down to $0.4 \%$ with total parotidectomy [2]. For this reason, superficial parotidectomy or total parotidectomy is generally preferred over enucleation.

Pleomorphic adenoma of the parotid gland usually presents as a single mass at initial presentation. In contrast, recurrent masses present as multinodular lesion in 33\%-98\% of cases. Recurrent tumors usually appear near the surgical scar from previous resection. Recurrence rates do not vary according to age or gender. An incomplete resection of adenoma could be due to capsular penetration that leads to the spread of tumor cells or to the existence of satellite lesions surrounding the capsule. One strategy to reduce the rate of recurrence is to re-evaluate surgical margins using intraoperative frozen sections. In addition, the optimal regimen should involve total parotidectomy, rather than enucleation or superficial parotidectomy $[1,2,4]$.

Zernial et al. [5] reported that most recurrent adenomas are located laterally to the facial nerve and that patients present with recurrent mass at an average of 8.1 years following the initial resection. In the same study, however, the authors reported a case, in which pleomorphic adenoma had recurred in 17 months of initial operation. Regular ultrasound monitoring is a prudent postoperative practice for patients who received local resection or had risk factors such as multinodular masses $[1,5]$.

Although complete resection of the parotid gland decreases the risk of recurrence, the major disadvantage is with the potential complication for facial nerve damage. Scarring of the surrounding tissues results in anatomical changes that make it difficult to distinguish between tumor and normal tissue. In many cases, the fa- cial nerve is surrounded by scar tissue [2,4]. For repeated resection of recurrent tumors, facial paralyses is present in about $51 \%$ of patients, with most recovering facial expressions within 1 year. Up to $23 \%$ of facial paralyses were found to be permanent [4].

Radiation therapy may be an option for postoperative treatment of recurrent pleomorphic adenoma, especially for cases in which multinodular masses were present, multiple recurrences have occurred, facial nerves must be sacrificed, or complete excision is practically difficult. However, no strong evidence exists for efficacy of radiation therapy, and some authors have suggested that radiation therapy should be kept as the last resort management of malignant transformation $[1,3]$.

The patient experienced repeated recurrence of pleomorphic adenoma despite multiple resections. Because of the adhesion between the tumor and facial nerve, the patient suffered from postoperative facial nerve damage. However, we observed only minor facial nerve weakening at 18 -month follow-up. While multiple recurrence of pleomorphic adenoma has been reported elsewhere, our patient represents the first report of such a case in the Korean population [2]. Recurrence within 17 months of initial operation is rare for pleomorphic adenoma, and our patient demonstrates that even a complete parotidectomy may be inadequate in preventing recurrence in certain cases.

\section{REFERENCES}

1. Redaelli de Zinis LO, Piccioni M, Antonelli AR, Nicolai P. Management and prognostic factors of recurrent pleomorphic adenoma of the parotid gland: personal experience and review of the literature. Eur Arch Otorhinolaryngol 2008;265:447-52.

2. Wittekindt C, Streubel K, Arnold G, Stennert E, Guntinas-Lichius O. Recurrent pleomorphic adenoma of the parotid gland: analysis of 108 consecutive patients. Head Neck 2007;29:822-8.

3. Mendenhall WM, Mendenhall CM, Werning JW, Malyapa RS, Mendenhall NP. Salivary gland pleomorphic adenoma. Am J Clin Oncol 2008;31:95-9.

4. Zbaren P, Tschumi I, Nuyens M, Stauffer E. Recurrent pleomorphic adenoma of the parotid gland. Am J Surg 2005;189:203-7.

5. Zernial O, Springer IN, Warnke P, Harle F, Risick C, Wiltfang J. Longterm recurrence rate of pleomorphic adenoma and postoperative facial nerve paresis (in parotid surgery). J Craniomaxillofac Surg 2007;35:189-92. 